\title{
THE ECONOMIC BURDEN OF FRAILTY AMONG ELDERLY PEOPLE: A REVIEW OF THE CURRENT LITERATURE
}

\author{
Alkhodary A. A. ${ }^{1,5}$, Syed Mohamed Aljunid ${ }^{1,2}$, Ismail A. ${ }^{3}$, Nur A. M. ${ }^{2}$ and Shahar S. ${ }^{4}$ \\ ${ }^{1}$ International Centre for Casemix and Clinical Coding, Faculty of Medicine, National University of Malaysia \\ 2 Department of Health Policy and Management, Faculty of Public Health, Kuwait University, Kuwait \\ ${ }^{3}$ Department of Community Health, Faculty of Medicine, National University of Malaysia \\ ${ }^{4}$ Programme of Dietetics, Faculty of Health Science, National University of Malaysia \\ ${ }^{5}$ Ministry of Health, Gaza Governorates, Palestine
}

Corresponding address: Ismail A.

Email: aniza@ppukm.ukm.edu.my

\begin{abstract}
Life expectancy from birth is increasing dramatically. Due to this increase, the population of elderly people will increase. Consequently, geriatric related illnesses will increase leading to increased necessity to build up comprehensive and coordinated cost effective health care services appropriate for elderly people. Frailty is not a disease, but rather considered as a syndrome requiring comprehensive and multidisciplinary care approach. It is a prevalent reversible pathological transitional stage between healthy aging and disability. Frailty is associated significantly with increased health care utilization, mortality, and comorbidities such falls, hospitalizations, physical dependence, and poor perception of health. The aim of this review is to compile existing literature on the economic cost of frailty syndrome among elderly people in the recent years. Search queries were constructed to look for articles related to the economic cost of frailty in the electronic databases available at the National University of Malaysia library for articles published between the years 2011 and 2019. The accessed electronic database included New England journal of medicine, Science Direct, SCOPUS, BMJ, Cochrane, and Wiley Online Library. Articles included in this review when they were original research, participants were defined as frail elderly, manuscripts written in English language, and involved clearly described measures of frailty cost. Among the literature, twenty one articles were found to satisfy the inclusion criteria of the review process. The cost of care for frail elderly was ranging from US $\$ 8,620$ to 29,910 per patient per year. The cost of health care was ranging from US $\$ 2,540$ to 221,400. The health care cost was accounting for $40 \%$ to $76 \%$ of the total care cost. Hospitalization cost was the highest, it was ranging from US $\$ 806$ to 152,726 . Outpatient cost was ranging from US $\$ 200$ to 18,000. Medications cost was ranging from US $\$ 7$ to 3,434 per frail elderly patient per year. Home help cost was ranging from US $\$ 804$ to 19,728 per frail elderly patient per year. In conclusion, frailty is a costly syndrome. It can be considered as a cost effective target for health promoting interventions to contain future elderly cost.
\end{abstract}

Key words: Frail, Frailty, Elderly, Economic, Cost, Burden

\section{INTRODUCTION}

As a result of advancement of the health care services and expanded human access to it, life expectancy from birth is increasing dramatically [1]. Globally, life expectancy of humans has increased from 61.7 years in 1980 to 71.8 years in 2015 [2, 3]. Due to this increase, the population of elderly people will increase [2]. The rate of population increase will be faster and pronounced among elderly people aged 60 years and over more than in any other age groups [1]. In the year 2017, the people aged 60 years or over comprised $13 \%$ of the global human population with a growing rate of $3 \%$ annually [2]. Additionally, the global number of elderly people aged 60 years and over is expected to increase from 900 millions in the year 2015 to 2 billions in the year 2050 [2, 4]. As a consequence, the percentage of geriatric related illnesses will increase leading to increased necessity to build up comprehensive and coordinated health care facilities and efficient services appropriate for elderly people's needs [5]. Globally, the current expenditure on health as \% of Gross Domestic Product was $6.3 \%$ in the year 2015 and was US $\$ 822$ per capita in the same year [6].

Frailty is not a disease, but rather considered as a syndrome requiring a comprehensive and multidisciplinary care approach, it is a reversible pathological transitional stage between healthy aging and disability [7-13]. Across the studies, frailty prevalence had a wide interval limit $[9,14$ 16]. The prevalence of frailty among elderly people is ranging from $4 \%$ to $59.1 \%$ depending on the adopted operational definition of frailty and the characteristics of the studied population [9]. This syndrome is associated significantly with increased health care utilization, mortality and comorbidities such falls, hospitalizations, physical dependence and poor perception of health $[9,12$, 15, 17]. Additionally, frail elderly are characterized by reduced reserve to resist any decline or impairment in respiratory, cardiovascular, renal, and cognitive functions [10, 18]. Moreover, frailty syndrome had a negative linear relationship with health related quality of life $[9,19]$. 
In order to understand the meaning of the frailty term properly, it is worth mentioning that several defining tests were used among the literature [9, $13,16,18,20]$. Fried's phenotype test was used to define frailty in the majority of studies [13, 15, 18, 20, 21]. According to Fried, Tangen [22], frailty is defined as a clinical syndrome constituted by the co-occurrence of at least three of the following five criteria: unintentional weight loss; exhaustion; weakness; slow walking speed; and low physical activity. Among elderly people, and as a result of increased health care needs mentioned above, frailty can be considered as one of the health care cost drivers for health care systems. The health care costs associated with frailty can be calculated by using two main approaches, a top down and bottom up costing methods [23]. The selection of the appropriate costing approach is dependent on the type and availability of data in the health care facility. According to Drummond, Sculpher [23], the driven cost of care can be classified into direct and indirect costs; the direct cost results from the direct utilization of the health care services and accompanying payments, while the indirect cost results from the lost productivity of the patients or patient's relatives and lost quality adjusted life years (QALY). In this review article, the focus will be on discussing the available literatures on the direct cost of frailty among elderly.

The purpose of this narrative review article is to compile existing literature on the economic cost of frailty syndrome among elderly people, to identify weaknesses and strengths permeated in the calculation process in recent years in order to improve the quality and precision of the measurement process in the future. Although, to recommend better ways of cost calculation in the future. To the best of the authors' knowledge, this study, which focuses specifically on the economic cost of frailty among elderly people is unprecedented.

\section{METHODS}

Selected keywords (Elderly, Frail, Frailty, Economic, Cost, Burden) were used to construct several searching queries for articles related to the economic cost of frailty in the electronic databases available at the National University of Malaysia library (Universiti Kebangsaan Malaysia -
UKM). These databases included EBSCOHOS, Ovid, Nature.com, Jaypeedigital, New England journal of medicine, Science Direct, SCOPUS, BMJ, Cochrane, and Wiley Online Library. The search process was performed to look for articles published between the years 2011 and 2019 . Articles were included if they met the following criteria: original articles; study participants were defined as frail elderly; full text of the article written in English language; Involving a clearly described figures on the economic cost of frailty for elderly people regardless their age; using any available frailty definition among the literature. The search process ended on April 20, 2019.

A total of 3560 articles were identified through the whole searching process of all queries. Excluded duplicate articles were 365 in the first stage of search. The second stage of search was to do screening for relevancy of the titles, abstracts, and key words among the 3204 filtered articles. A total of 95 articles were found relevant to the current review title. The third stage was to review full text of the manuscripts looking for frailty cost figures. A total of 21 articles were found satisfying the inclusion criteria of the study, as mentioned above. All excluded articles were either not including any frailty cost figures or written in languages other than English.

Full texts of the final 21 articles were reviewed and data were extracted. MS Excel (Version 2013) was used to tabulate extracted data from these articles. Figure 1 presents the flow chart of articles selection process. Pivot tables were used to check for and build up new tables and figures. Data extraction process was primarily focused on study design, methodology of sampling, adopted frailty definition, frailty cost findings, and other related variables (table 1 in Appendix). Within the interventional trials $(n=11)$, frailty cost was extracted from the control groups. For all studies, currency used and duration of study were standardized to facilitate comparisons. All cost figures of frailty were standardized into one year cost. Additionally, standardization was done for the currency into US Dollars. It is worth mentioning that the reported cost figures of frail elderly people were including health care needs for all comorbidities of the patients during the specified durations of studies. 


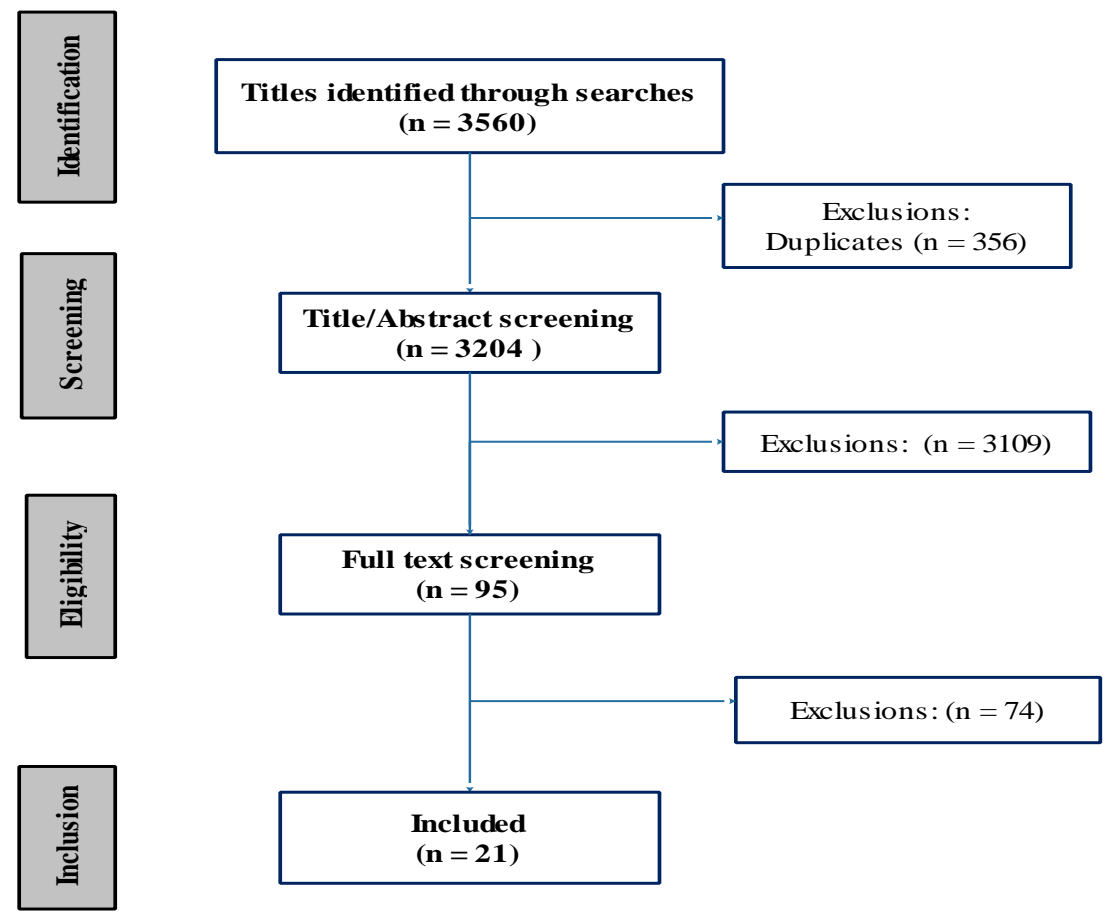

(Figure 1) : Articles selection process

\section{RESULTS}

Figure 2 shows the distribution of articles among countries conducted the studies, a total of 21 articles were included in this systematic review. It was found that the majority of studies came from Europe $(n=15)$, while the remaining studies came from USA $(n=4)$, and Australia $(n=2)$. Netherland was on the top rank in a number of studies conducted to quantify the economic cost of frailty syndrome. Table 1 summarizes the articles included in the current review paper. Regarding the time approach of summarized articles, it was found that the majority of the articles used a prospective approach $(n=16)$, while the remaining used a retrospective approach. With regard to the years of conducting and publishing the studies, it was found that the analyzed data of these articles were collected between the years 2002 and 2015. All studies were published between the years 2011 and 2018, the majority of these articles were published between 2015 and $2017(n=15)$. Several types of studies were used to identify the cost of frailty: cross-sectional $(n=7)$, cohort studies $(n=3)$, randomized clinical trials $(\mathrm{RCT}, \mathrm{n}=8)$, nonrandomized clinical trial $(n=1)$, and quasi experimental study $(n=1)$ (Table 1 in appendix).

The type of data collection tools used among the studies were also various (e.g. Questionnaire, Physical assessment, Database screening). The majority of the studies used at least two data collection tools ( $n=16)$, questionnaires were used alone or in combination with other tools in the majority of studies $(n=17)$. The age of the study participants was variable also; the majority $(n=$ 15 ) of the studies adopted a minimum age of 65 years as a selection criteria (8 studies selected participants aged 65 years or older, 5 studies selected participants aged 70 years or older, 4 studies selected participants aged 75 years or older). Concerned with the adopted test for defining frailty among the study participants, it was found that more than ten different tests of frailty syndrome were used to detect frail participants, Fried 's test of frailty (also known as CHS or frailty phenotype) and Groningen Frailty Indicator (GFI) were more commonly used. The currency used to quantify the cost figures of frailty was also varying according to the country of the study: 12 studies used Euro currency; 6 studies used US dollar currency; while 2 studies used Australian dollar currency; and only one study used pound sterling currency to quantify the health care cost for frail elderly. The duration of calculated cost of frailty syndrome was varying among the studies. The most commonly used duration to measure the cost of frailty syndrome was one year period $(n=10)$; the minimum duration used to measure the cost of frailty syndrome was three months $(n=5), 6$ months duration was used in 4 studies, 9 months and 24 months duration were used by only one study for each duration period. 


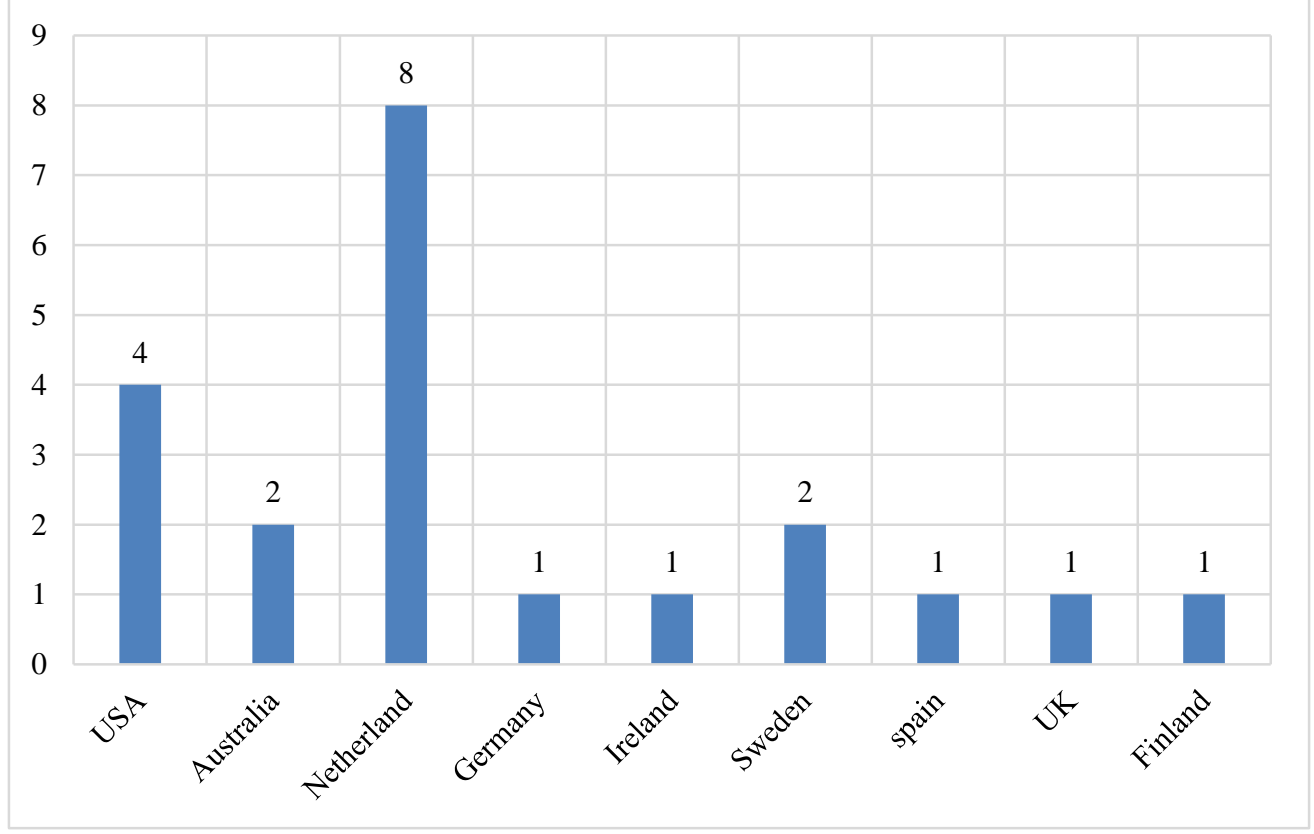

Figure 2 : The distribution of articles among countries

Table 2 summarizes the cost figures of health care services provided for frail elderly reported in the included articles. It was found that the vast majority of the studies $(n=19)$ included figures about the total cost of health care services provided for frail patients, while only one third ( $\mathrm{n}$ $=8$ ) of the studies reported figures on the total cost of care. Among the studies, the total cost of care was widely variable, it was ranging from US $\$ 8,620$ to US $\$ 29,910$ per frail elderly patient per year. A Netherland study [24] reported the lowest cost of care services for frail elderly patients, while an Australian study [25] reported the highest cost of care services for frail elderly patients. Additionally, the total cost of health care services was also widely variable among the studies, it was ranging from US $\$ 2,540$ to US $\$$ 221,400 per frail elderly patient per year. A Netherland study [26] reported the lowest cost of health care for frail elderly patients, while a USA study [27] reported the highest cost of health care for frail elderly patients. Apparently, the majority of the studies revealed that the health care cost for frail elderly was between US $\$ 7,100$ to US $\$$ 70,000 per patient per year.

Concerning the cost figures of health care provided for frail elderly, it was found that the total hospitalization cost ranging from US $\$ 806$ to US $\$ 152,726$ per patient per year. Two Netherland studies $[28,29]$ reported the lowest hospitalization cost, while a USA study [27] and another Sweden study [30] reported the most expensive cost for hospitalization services providers for frail elderly patients. In connection with the cost of outpatient services, only half of the studies quantified the cost of outpatient care services, the outpatient cost was ranging from US $\$ 200$ to US $\$ 18,000$ per frail elderly patient per year. Two Netherland studies $[28,29]$ and one Sweden study [30] reported the lowest cost of outpatient care for frail elderly patients, while one USA study [31] reported the highest cost of outpatient care services for frail elderly patients. Additionally, half of the studies measured the cost of general practitioners services. The calculated cost for general practitioners service was ranging from US $\$ 278$ to US $\$ 12,729$ per patient per year; three Netherland studies [28, 29, 32] reported the lowest cost of general practitioners services for frail elderly patients, while one USA study [33] reported the highest cost of general practitioners service for frail elderly patients.

A total of seven articles reported the cost of medications for frail elderly patients. The medication cost was ranging from US $\$ 7$ to US $\$$ 3,434 per frail elderly patient per year. Two Netherland studies [26, 32] reported the lowest cost of medications for frail elderly patients, while one USA study [31] reported the highest cost of medications for frail elderly patients. These medications included all patient needs during the studied durations. As for the cost of home help services for frail elderly patients, it was found that 8 studies calculated the cost of this item, five of it were Netherland studies. The cost of home help services for frail elderly patients was ranging from US $\$ 804$ to 19,728 per frail elderly patient per year. An Australian study [25] reported the lowest cost of home help services for frail elderly patients, while a Netherland study [26] reported the highest home help cost for frail elderly patients. In addition, nursing care cost was calculated in 6 studies only. The cost of nursing care services for frail elderly patients was ranging from US $\$ 349$ to US $\$ 9,003$ per patient per year. A Sweden study [34] reported the lowest nursing care cost for frail elderly patients, while a Netherland study [28] reported the highest nursing care cost for frail elderly patients. Moreover, residential care cost 
was calculated in 7 studies. The cost of residential care services for frail elderly patients was ranging from US $\$ 844$ to US $\$ 52,878$ per patient per year. An Australian study [25] reported the lowest cost of residential care services for frail elderly patients, while a Netherland study [26] reported the highest residential care cost for frail elderly patients. Furthermore, informal care cost was calculated in 7 studies. The cost of informal care services for frail elderly patients was ranging from US $\$ 2,246$ to US $\$ 41,113$ per patient per year. A Netherland study [24] reported the lowest informal care cost for frail elderly patients, while an Irish study [35] reported the highest informal care cost for frail elderly patients.

\section{DISCUSSION}

The current systematic review aims to put into perspective the updated trends of economic cost of frailty among elderly people as a health care cost driver globally. The concept of measuring the economic cost of frailty syndrome seems to be new, since only 21 articles found on the cost of such syndrome. Additionally, as a rising public health issue, it is gaining more interest in the recent few years. It was noticed that 12 studies related to this topic were published between 2016 and 2018. This reflects the fact that as humans age, elderly people are increasing, and elderly related needs are more considered as an important pressing issue in communities. The studies showed that European countries are more interested in calculating the economic cost of this syndrome. This can be considered as logic, because of the fact that Europe is the most aged continent in terms of the proportion of elderly people, as $25 \%$ of the total population of Europe are aged 60 years and over [2]. Data collection tools were also different among the studies, this can be a result of variations in the health care system components, different health record formats, and different data sources among the different countries. In order to identify the best among these data collection tools, the researchers see that it is necessary to compare detailed content of these tools to identify strengths and weaknesses in it and to recommend one as the most sensitive tool to be used in future costing studies. This can make it possible to do full cost comparisons among frail elderly patients of different countries and make it easier to discover and interpret new findings. The findings of this review article clearly shows that different durations were used to calculate the economic cost of frailty; this variation might be due to varied amounts of funds allocated for each study or can be due to repeated emergence of the same findings after a certain time of the study. It was noticed that both Netherland and USA studies used three different cost durations within each country. The researchers see that calculating the cost of frailty syndrome for one full year is the best option. This opinion can be justified by the assumption that one year duration gives the opportunity to calculate the cost of health care utilization during four different seasons. It is well known that elderly health care needs are variable during different seasons of the year. Findings of this review article showed that different tests were used to detect frailty syndrome among elderly patients, which means that case matching criteria were not fully achieved. Additionally, participants' comorbidities were not clearly unique among the reviewed studies. These might be important rationale for observed variations in the economic cost of frailty syndrome among elderly. Data collectors were also varying among the studies; the most commonly used data collectors were GPs and nurses. Some studies included physiotherapists, occupational therapists, dietitians, and administrative staff as data collectors, while others did not mention the data collection team qualifications at all.

Findings of this review article showed that several health care service terms were used to describe the components of the health care cost provided for frail elderly patients. These mentioned terms included hospitalization, outpatient care, medications, emergency services, general practitioners, nursing care, residential care, home help, community services, total health care, and total care. Probably, these different terms resulted from differences among countries' health care system components and different health care services provided in each health care system. Additionally, it was not clear if the cost items not mentioned in the articles were included under other items of care or not. As a result of that, it was difficult to make a clear cost comparison between different health care systems that has different components and different provided care services. Hospitalization and total health care costs were the most commonly used terms among the studies. In addition, home help, residential care, and informal care represented the remaining costly mentioned care services provided for frail elderly patients. Notably, these cost items were not mentioned in many studies.

It was found that health care cost accounting for $40 \%$ to $76 \%$ of the total care cost provided for frail elderly patients. More specifically, hospitalization cost was the highest among the health care cost items of frail elderly. A published statistics showed that the hospital length of stay indicator among these countries is not too much different to justify these wide variations in the hospitalization cost. The minimum hospital length of stay was reported in Australia with an average of 4.2 hospitalization days, while the maximum hospital length of stay was reported in Germany with an average of 7.5 hospitalization days [36]. That means, hospitalization cost wide variation cannot be justified by variations in the hospital length of stay reported among these countries. Probably, these cost variations might be 
attributable to differences in years of conducting the studies and variations in health care system components of different countries. One more important factor that can affect the hospitalization cost of frailty is the comorbidities among frail patients. It is well known that hospitalization needs are increased with comorbidities dramatically. Also, age of study participants was different among the studies as mentioned above. It is well known that advanced age is associated with increased comorbidities and health care needs, this can be considered as another justification for the observed variations in frailty cost. Additionally, it was noticed that sampling criteria and sample size of the studies were different among the reviewed studies. It is well known that both sampling criteria and sample size of the study can affect health care cost findings. Another justification for health care cost variation among the studies could be due to variations in the nature, content, quality, and relative prices of health care services provided in different countries. It is well known that all these factors are different among different countries. An additional explanation for health care cost variation among frail elderly can be attributable to differences in health care spending as a \% of GDP in different countries. The USA spent about $17.2 \%$ of GDP on health during the year 2016, while European countries spent an average of $8 \%$ to $11 \%$ of GDP on health in the same year [36]. Additionally, in order to reduce the gap of health care cost variations, the researchers saw that both findings of Robinson, Wu [27] and Overbeek, Polinder [26] cost values were extreme outliers of frail elderly health care cost when compared to other studies. In order to illustrate that, it is worth mentioning that there were three other American studies that found health care costs of frail elderly patients ranging from US \$ 10,700 to US $\$ 70,100$ per patient per year, which is less than half of the cost in Robinson, Wu [27] study. Moreover, there were three Netherland studies found the health care cost of frail elderly patients ranging from US $\$ 18,675$ to US $\$ 34,927$ per patient per year, which is seven times more than the cost observed in Overbeek, Polinder [26] study.

Limitations of our review are scarce number of relevant articles, use of various frailty tests for detection of patients in different studies, use of different health care service terms for description of frailty cost, use of different age groups as an inclusion criteria, conducting the studies in different years, and use of various time durations among the conducted studies as well. Additionally, the comorbidities criteria were not clearly standardized. However, the main scope of this systematic review was to evaluate the current available economic cost of frailty syndrome among elderly people. A strength of this review is that our literature search identified all frailty cost published until April 2019. Consequently, our results reflect the present situation regarding the economic cost of frailty among elderly worldwide. Our integrative review revealed that frailty is a costly phenomena. In summary, no single costing structure was used to calculate the cost of frailty syndrome among elderly, many different definitions and tests of frailty syndrome were used to detect the cases in different studies, a wide range of total and health care cost of frailty syndrome were noticed.

\section{CONCLUSION}

Consequently, we can conclude that frailty is a costly syndrome that justify specific research focus. Hospitalization was the most costly part of health care services provided for frail elderly patients. Further studies are needed to draw a clear distributive mapping for the cost of frail elderly among health care services. Probably, this can be considered as the first step in developing comprehensive and cost efficient care services for this group of people. Additionally, frailty can be considered as a cost effective target for interventions to contain future care cost. It is not clear what sociodemographic and co-morbidity factors that affect the cost of care more among frail patients. Furthermore, the patterns of health care utilization of frail elderly among different age groups need to be studied. Finally, different perspectives of the cost of frailty (providers, societal, carer) need to be addressed as well.

\section{REFERENCES}

1. WHO, W.H.O., Active ageing: A policy framework. 2002, Geneva: World Health Organization.

2. UN, U.N., World population prospects: The 2017 revision. United Nations Econ Soc Aff, ed. W.P.N. ESA/P/WP/248. 2017, New york: United Nations.

3. Wang, H., et al., Global, regional, and national life expectancy, allcause mortality, and cause-specific mortality for 249 causes of death, 1980-2015: a systematic analysis for the Global Burden of Disease Study 2015. The lancet, 2016. 388(10053): p. 1459-1544.

4. WHO, W.H.O., Elder abuse fact sheet. Website:

http://www.who.int/en/newsroom/fact-sheets/detail/elder-abuse Accessed October, 22, 2018.

5. WHO, W.H.O., World report on ageing and health. 2015: World Health Organization. 
6. WHO, World health statistics 2018: monitoring health for the SDGs, sustainable development goals. 2018.

7. Boyle, P.A., et al., Physical frailty is associated with incident mild cognitive impairment in communitybased older persons. Journal of the American Geriatrics Society, 2010. 58(2): p. 248-255.

8. Butts, B. and R. Gary, Coexisting frailty, cognitive impairment, and heart failure: implications for clinical care. Journal of clinical outcomes management: JCOM, 2015. 22(1): p. 38.

9. Cesari, M., et al., Frailty: an emerging public health priority. Journal of the American Medical Directors Association, 2016. 17(3): p. 188-192.

10. Clegg, A., et al., Frailty in elderly people. The lancet, 2013. 381(9868): p. 752-762.

11. Ferrer, A., et al., Frailty in the oldest old: prevalence and associated factors. Journal of the American Geriatrics Society, 2013. 61(2): p. 294-296.

12. Morley, J.E., et al., Frailty consensus: a call to action. Journal of the American Medical Directors Association, 2013. 14(6): p. 392-397.

13. Rodríguez-Mañas, L., et al., Searching for an operational definition of frailty: a Delphi method based consensus statement. The frailty operative definition-consensus conference project. Journals of Gerontology Series A: Biomedical Sciences and Medical Sciences, 2012. 68(1): p. 62-67.

14. Bieniek, J., K. Wilczyński, and J. Szewieczek, Fried frailty phenotype assessment components as applied to geriatric inpatients. Clinical interventions in aging, 2016. 11: p. 453.

15. Nguyen, T., R. Cumming, and $\mathrm{S}$. Hilmer, $A$ review of frailty in developing countries. The journal of nutrition, health \& aging, 2015. 19(9): p. 941-946.

16. Shamliyan, T., et al., Association of frailty with survival: a systematic literature review. Ageing research reviews, 2013. 12(2): p. 719-736.

17. Bherer, L., K.I. Erickson, and T. LiuAmbrose, A review of the effects of physical activity and exercise on cognitive and brain functions in older adults. Journal of aging research, 2013. 2013.

18. Halil, M., et al., Cognitive aspects of frailty: mechanisms behind the link between frailty and cognitive impairment. The journal of nutrition, health \& aging, 2015. 19(3): p. 276283.

19. Buck, H.G. and B. Riegel, The impact of frailty on health related quality of life in heart failure. European Journal of Cardiovascular Nursing, 2011. 10(3): p. 159-166.

20. Houles, M., et al., Frailty and cognition. J Frailty Aging, 2012. 1(2): p. 56-63.

21. Kulmala, J., et al., Association between frailty and dementia: a population-based study. Gerontology, 2014. 60(1): p. 16-21.

22. Fried, L.P., et al., Frailty in older adults: evidence for a phenotype. The Journals of Gerontology Series A: Biological Sciences and Medical Sciences, 2001. 56(3): p. M146-M157.

23. Drummond, M.F., et al., Methods for the economic evaluation of health care programmes. 2015: Oxford university press.

24. Bleijenberg, N., et al., CostEffectiveness of a Proactive Primary Care Program for Frail Older People: A Cluster-Randomized Controlled Trial. Journal of the American Medical Directors Association, 2017. 18(12): p. 1029-1036. e3.

25. Fairhall, N., et al., Economic Evaluation of a Multifactorial, Interdisciplinary Intervention Versus Usual Care to Reduce Frailty in Frail Older People. Journal of the American Medical Directors Association, 2015. 16(1): p. 41-48.

26. Overbeek, A., et al., Advance Care Planning for frail older adults: Findings on costs in a cluster randomised controlled trial. Palliative medicine, 2018: p. 0269216318801751. 
27. Robinson, T.N., et al., Frailty predicts increased hospital and sixmonth healthcare cost following colorectal surgery in older adults. The American Journal of Surgery, 2011. 202(5): p. 511-514.

28. Makai, P., et al., Cost-effectiveness of integrated care in frail elderly using the ICECAP-O and EQ-5D: does choice of instrument matter? The European journal of health economics, 2015. 16(4): p. 437-450.

29. Looman, W.M., et al., Costeffectiveness of the 'Walcheren Integrated Care Model'intervention for community-dwelling frail elderly. Family practice, 2016. 33(2): p. 154160.

30. Ekerstad, N., et al., Short-term Resource Utilization and CostEffectiveness of Comprehensive Geriatric Assessment in Acute Hospital Care for Severely Frail Elderly Patients. Journal of the American Medical Directors Association, 2018.

31. Simpson, K.N., et al., Effect of frailty on resource use and cost for Medicare patients. Journal of comparative effectiveness research, 2018(0).

32. de Vries, N.M., et al., Patient-centred physical therapy is (cost-) effective in increasing physical activity and reducing frailty in older adults with mobility problems: a randomized controlled trial with 6 months followup. Journal of cachexia, sarcopenia and muscle, 2016. 7(4): p. 422-435.

33. Joynt, K.E., et al. Segmenting highcost Medicare patients into potentially actionable cohorts. in Healthcare. 2017. Elsevier.

34. Sandberg, M., et al., Cost-utility analysis of case management for frail older people: effects of a randomised controlled trial. Health economics review, 2015. 5(1): p. 12.

35. Butler, A., et al., Frailty: a costly phenomenon in caring for elders with cognitive impairment. International journal of geriatric psychiatry, 2016. 31(2): p. 161-168.

36. OECD. OECD Data Indicators, Length of hospital stay. . OECD Publishing, Paris. DOI: 10.1787/8dda6b7a-en.
Accessed December, 13, 2018. 2018 [cited 11 ].

37. Peters, L.L., et al., Predictive validity of a frailty measure (GFI) and a case complexity measure (IM-E-SA) on healthcare costs in an elderly population. Journal of Psychosomatic Research, 2015. 79(5): p. 404-411.

38. Bock, J.-O., et al., Associations of frailty with health care costs-results of the ESTHER cohort study. BMC health services research, 2016. 16(1): p. 128.

39. Ensrud, K.E., et al., Frailty Phenotype and Healthcare Costs and Utilization in Older Women. Journal of the American Geriatrics Society, 2018.

40. van Leeuwen, K.M., et al., CostEffectiveness of a Chronic Care Model for Frail Older Adults in Primary Care: Economic Evaluation Alongside a Stepped-Wedge ClusterRandomized Trial. Journal of the American Geriatrics Society, 2015. 63(12): p. 2494-2504.

41. Tanajewski, L., et al., Costeffectiveness of a specialist geriatric medical intervention for frail older people discharged from acute medical units: economic evaluation in a two-centre randomised controlled trial (AMIGOS). PloS one, 2015. 10(5): p. e0121340.

42. Comans, T.A., et al., The increase in healthcare costs associated with frailty in older people discharged to a post-acute transition care program. Age and ageing, 2016. 45(2): p. 317320.

43. Kehusmaa, S., et al., Factors associated with the utilization and costs of health and social services in frail elderly patients. BMC health services research, 2012. 12(1): p. 204.

44. Metzelthin, S.F., et al., Reducing disability in community-dwelling frail older people: cost-effectiveness study alongside a cluster randomised controlled trial. Age and ageing, 2015. 44(3): p. 390-396.

45. García-Nogueras, I., et al., Use of health resources and healthcare costs associated with frailty: the FRADEA 
study. The journal of nutrition, health \& aging, 2017. 21(2): p. 207-214. 